\title{
The Vernam cipher is robust to small deviations
}

\section{from randomness}

\author{
Boris Ryabko \\ Siberian State University of Telecommunications and Information Sciences, \\ Institute of Computational Technology of Siberian Branch of Russian \\ Academy of Science, Novosibirsk, Russia \\ boris@ryabko.net
}

\begin{abstract}
The Vernam cipher (or one-time pad) has played an important rule in cryptography because it is a perfect secrecy system. For example, if an English text (presented in binary system) $X_{1} X_{2} \ldots$ is enciphered according to the formula $Z_{i}=\left(X_{i}+Y_{i}\right) \bmod 2$, where $Y_{1} Y_{2} \ldots$ is a key sequence generated by the Bernoulli source with equal probabilities of 0 and 1 , anyone who knows $Z_{1} Z_{2} \ldots$ has no information about $X_{1} X_{2} \ldots$ without the knowledge of the key $Y_{1} Y_{2} \ldots$ (The best strategy is to guess $X_{1} X_{2} \ldots$ not paying attention to $Z_{1} Z_{2} \ldots$.)

But what should one say about secrecy of an analogous method where the key sequence $Y_{1} Y_{2} \ldots$ is generated by the Bernoulli source with a small bias, say, $P(0)=0.49, P(1)=0.51$ ? To the best of our knowledge, there are no theoretical estimates for the secrecy of such a system, as well as for the general case where $X_{1} X_{2} \ldots$ (the plaintext) and key sequence are described by stationary ergodic processes. We consider the runningkey ciphers where the plaintext and the key are generated by stationary
\end{abstract}


ergodic sources and show how to estimate the secrecy of such systems.

In particular, it is shown that, in a certain sense, the Vernam cipher is robust to small deviations from randomness.

Keywords: running-key cipher, Vernam cipher, Shannon entropy, unconditional secrecy.

\section{Introduction}

We consider the classical problem of transmitting secret messages from Alice (a sender) to Bob (a receiver) via an open channel which can be accessed by Eve (an adversary). It is supposed that Alice and Bob (and nobody else) know a so-called key $K$ which is a word in a certain alphabet. Before transmitting a message Alice encrypts it. In his turn, Bob, after having received the encrypted message (ciphertext), decrypts it to recover the initial text (plaintext).

We consider so-called running-key ciphers where the plaintext $X_{1} \ldots X_{t}$, the key sequence $Y_{1} \ldots Y_{t}$ and ciphertext $Z_{1} \ldots Z_{t}$ belong to one alphabet $A$ (without loss of generality we suppose that $A=\{0,1, \ldots, n-1\}$, where $n \geq 2$. The $i-t h$ letter of the ciphertext is defined by $Z_{i}=c\left(X_{i}, Y_{i}\right), \quad i=1, \ldots, t$, whereas the deciphering rule is by $X_{i}=d\left(Z_{i}, Y_{i}\right), i=1, \ldots, t$, i.e. $d\left(e\left(X_{i}, Y_{i}\right), Y_{i}\right)=X_{i}$. Here $c$ and $d$ are functions called coder and decoder, correspondingly. Quite often the following particular formula are used

$$
Z_{i}=\left(X_{i}+Y_{i}\right) \bmod n, \quad X_{i}=\left(Z_{i}-Y_{i}\right) \bmod n,
$$

i.e. $c\left(X_{i}, Y_{i}\right)=\left(X_{i}+Y_{i}\right) \bmod n, d\left(Z_{i}, Y_{i}\right)=\left(Z_{i}-Y_{i}\right) \bmod n$. In a case of two-letter alphabet (1) can be presented as follows:

$$
Z_{i}=\left(X_{i} \oplus Y_{i}\right), X_{i}=\left(Z_{i} \oplus Y_{i}\right)
$$


where $a \oplus b=(a+b) \bmod 2$.

It is important to note that we consider a so-called unconditional (or informationtheoretical) security. That is, the cipher is secure even when Eve has unlimited computing power. Roughly speaking, if the unconditionally secure cipher is used, Eve has many highly probable possible versions of a plaintext and, hence, cannot choose the real plaintext from them. The following informal consideration helps to understand the main idea of an approach considered later: Let there be two unconditionally secure ciphers which can be applied to one plaintext. Imagine, that for the first cipher Eve has 10 equiprobable possible deciphering texts whose overall probability equals 0.999 , whereas for the second cipher there are 100 equiprobable deciphering texts with the same overall probability. Obviously, the second system is more preferable, because the uncertainty of Eve is much larger for the second system. This informal consideration is quite popular in cryptography [6, 7, 9] and we will estimate the security of a cipher by the logarithm of the total number of (almost) equiprobable possible deciphering texts whose overall probability is close to 1 .

The running-key cipher (11) is called the Vernam cipher (or one-time pad) if any word $k_{1} \ldots k_{t}, k_{i} \in A$, is used as the key word with probability $n^{-t}$, i.e. $P\left(Y_{1} \ldots Y_{t}=k_{1} \ldots k_{t}\right)=n^{-t}$ for any $k_{1} \ldots k_{t} \in A^{t}$. In other words, we can say that the key letters are independent and identically distributed (i.i.d.) and probabilities of all letters are equal.

The Vernam cipher is one of the most popular among the unconditionally secure running-key ciphers. It has played an important rule in cryptography, especially since C.Shannon proved that this cipher is perfectly secure 9]. That is, the ciphertext $Z_{1} \ldots Z_{t}$ gives absolutely no additional information about the plaintext $X_{1} \ldots X_{t}$. This fact can be interpreted as follows: a priori probability of a plaintext is the same as a posteriori probability of a plaintext given the 
corresponding ciphertext [9]. Using Shannon entropy, it can be expressed by the following equation $h\left(X_{1} \ldots X_{t}\right)=h\left(X_{1} \ldots X_{t} \mid Z_{1} \ldots Z_{t}\right)$, where $h\left(X_{1} \ldots X_{t}\right)$ and $h\left(X_{1} \ldots X_{t} \mid Z_{1} \ldots Z_{t}\right)$ are the entropy of the plaintext and the conditional entropy of the plaintext given the ciphertext $Z_{1} \ldots Z_{t}$, correspondingly (they will be defined below). For example, if one uses the Vernam cipher (2) to cipher an English text presented, say, in standard 7-bit binary ASCII, Eve can try to guess the plaintext not paying attention on the ciphertext.

It was shown by Shannon that any perfectly secure system must use the secret key whose length equals the plaintext length. That is why many authors considered the problem of security of systems where either the length of the key or its entropy is less than the length (or entropy) of the plaintext, see, for example, [1, 4, 5, 6, 7, 9] and reviews therein. But, in spite of numerical papers, some seemingly natural questions are still open. For example, what can we say about secrecy of the system (2) where it is applied to an English text (in binary presentation) and the key sequence is generated by the Bernoulli source with a small bias, say, $P\left(Y_{i}=0\right)=0.51, P\left(Y_{i}=0\right)=0.49$. (Informally, it is "almost" Vernam cipher). To the best of our knowledge, there are no theoretical estimates for the security of such a system, as well as for the general case where the plaintext and key are described as stationary ergodic processes.

In this paper we consider this problem for running-key ciphers (1) in a case where the plaintext $X_{1} \ldots X_{t}$ and the key sequence $Y_{1} \ldots Y_{t}$ are independently generated by stationary ergodic sources and the entropy of the key can be less than maximally possible value $\log n$ per letter (here and below $\log \equiv \log _{2}$ ). The goal of the paper is to find simple estimates of secrecy for such systems. We would like to emphasize that the unconditional secrecy is meant, i.e. it is supposed that Eve has unlimited computational power and unlimited time for computations. 
It is worth noting that Shannon in his famous paper [9] mentioned that the problem of deciphering of a ciphertext and the problem of signal denoising are very close from mathematical point of view. In this paper we use some results obtained in [8] considering the problem of denoising.

\section{Preliminaries}

We consider the case where the plaintext $X=X_{1}, X_{2}, \ldots$ and and the key sequence $Y_{1}, Y_{2}, \ldots$ are independently generated by stationary ergodic processes with the finite alphabets $A=\{0,1, \ldots, n-1\}, n \geq 2$.

The $m$-order Shannon entropy and the limit Shannon entropy are defined as follows:

$$
h_{m}(X)=-\frac{1}{m+1} \sum_{u \in A^{m+1}} P_{X}(u) \log P_{X}(u), \quad h(X)=\lim _{m \rightarrow \infty} h_{m}(X)
$$

where $m \geq 0, P_{X}(u)$ is the probability that $X_{1} X_{2} \ldots X_{|u|}=u$ (this limit always exists, see, for ex., [2, 3]). Introduce also the conditional Shannon entropy

$$
h_{m}(X \mid Z)=h_{m}(X, Z)-h_{m}(Z), h(X \mid Z)=\lim _{m \rightarrow \infty} h_{m}(X \mid Z)
$$

The Shannon-McMillan-Breiman theorem for conditional entropies can be stated as follows.

Theorem 1 (Shannon-McMillan-Breiman). $\forall \varepsilon>0, \forall \delta>0$, for almost all $Z_{1}, Z_{2}, \ldots$ there exists $n^{\prime}$ such that if $n>n^{\prime}$ then

$$
P\left\{\left|-\frac{1}{n} \log P\left(X_{1} . . X_{n} \mid Z_{1} . . Z_{n}\right)-h(X \mid Z)\right|<\varepsilon\right\} \geq 1-\delta
$$

The proof can be found in $[2,3]$. 


\section{Estimations of secrecy}

Theorem 2. Let a plaintext $X=X_{1} X_{2}, \ldots$ and the key sequence $Y=Y_{1} Y_{2}, \ldots$ be stationary ergodic processes with a finite alphabet $A=\{0,1, \ldots, n-1\}, n \geq 2$, and let a running-key cipher be applied to $X$ and $Y$ and $Z=Z_{1}, Z_{2}, \ldots$ be the ciphertext. Then, for any $\varepsilon>0$ and $\delta>0$ there is such an integer $n^{\prime}$ that, with probability 1 , for any $t>n^{\prime}$ and $Z=Z_{1}, Z_{2}, \ldots Z_{t}$ there exists the set $\Psi(Z)$ for which the following properties are valid:

i) $P(\Psi(Z))>1-\delta$

ii) for any $X^{1}=X_{1}^{1}, \ldots, X_{t}^{1}, X^{2}=X_{1}^{2}, \ldots, X_{t}^{2}$ from $\Psi(Z)$

$$
P\left\{\frac{1}{t}\left|\log P\left(X^{1} \mid Z\right)-\log P\left(X^{2} \mid Z\right)\right|<\varepsilon\right\}
$$

iii) $\liminf _{t \rightarrow \infty} \frac{1}{t} \log |\Psi(Z)| \geq h(X \mid Z)$.

Proof. According to Shannon-McMillan-Breiman theorem for any $\varepsilon>0, \delta>0$ and almost all $Z_{1}, Z_{2}, \ldots$ there exists $\operatorname{such} n^{\prime}$ that for $t>n^{\prime}$

$$
P\left\{\left|-\frac{1}{t} \log P\left(X_{1} X_{2} \ldots X_{t} \mid Z_{1} Z_{2} \ldots Z_{t}\right)-h(X \mid Z)\right|<\varepsilon / 2\right\} \geq 1-\delta .
$$

Let us define

$$
\Psi(Z)=\left\{X=X_{1} X_{2} \ldots X_{t}: P\left(X_{1} X_{2} \ldots X_{t} \mid Z_{1} Z_{2} \ldots Z_{t}\right)-h(X \mid Z) \mid<\varepsilon / 2\right\} .
$$

The first property i) immediately follows from (6). In order to prove ii), note that for any $X^{1}=X_{1}^{1}, \ldots, X_{t}^{1}, X^{2}=X_{1}^{2}, \ldots, X_{t}^{2}$ from $\Psi(Z)$ we obtain from (6), (77)

$$
\frac{1}{t}\left|\log P\left(X^{1} \mid Z\right)-\log P\left(X^{2} \mid Z\right)\right| \leq \frac{1}{t}\left|\log P\left(X^{1} \mid Z\right)-h(X \mid Z)\right|
$$




$$
+\frac{1}{t}\left|\log P\left(X^{2} \mid Z\right)-h(X \mid Z)\right|<\varepsilon / 2+\varepsilon / 2=\varepsilon .
$$

From (7) and the property i) we obtain the following: $|\Psi(Z)|>(1-\delta) 2^{t(h(X \mid Z)-\varepsilon)}$. Taking into account that it is valid for any $\varepsilon>0, \delta>0$ and $t>n^{\prime}$, we obtain iii).

So, we can see that the set of possible decipherings $\Psi(Z)$ grows exponentially, its total probability is close to 1 and probabilities of words from this set are close to each other.

Theorem 2 gives a possibility to estimate an uncertainty of a cipher based on the conditional entropy $h(X \mid Z)$. Sometimes it can be difficult to calculate this value because it requires knowledge of the conditional probabilities. In this case the following simpler estimate can be useful.

Corollary 1. For almost all $Z_{1} Z_{2} \ldots$

$$
\liminf _{t \rightarrow \infty} \frac{1}{t} \log |\Psi(Z)| \geq h(X)+h(Y)-\log n .
$$

Proof. From the well-known in Information Theory equation $h(X, Z)=h(X)+$ $h(Z \mid X)$ (see [2, 3]) we obtain the following:

$$
h(X \mid Z)=h(X, Z)-h(Z)=h(Z \mid X)+h(X)-h(Z) .
$$

Having taken into account that $\max h(Z)=\log n([2,3)$, where $n$ is the number of alphabet letters, we can derive from the latest equation that $h(X \mid Z) \geq$ $h(Z \mid X)+h(X)-\log n$. The definition of the running-key cipher (10) shows that $h(Z \mid X)=h(Y)$. Taking into account two latest inequalities and the third statement iii) of Theorem 2 we obtain the statement of the corollary.

Comment. In Information Theory the difference between maximal value of the entropy and real one quite often is called the redundancy. Hence, from 
the corollary we have new following presentations for the value $\frac{1}{t} \log |\Psi(Z)|$ :

$$
\begin{gathered}
\liminf _{t \rightarrow \infty} \frac{1}{t} \log |\Psi(Z)| \geq h(X)-r_{Y}, \quad \liminf _{t \rightarrow \infty} \frac{1}{t} \log |\Psi(Z)| \geq h(Y)-r_{X}, \\
\liminf _{t \rightarrow \infty} \frac{1}{t} \log |\Psi(Z)| \geq \log n-\left(r_{X}+r_{Y}\right),
\end{gathered}
$$

where $r_{Y}=\log n-h(Y)$ and $r_{X}=\log n-h(X)$ are the corresponding redundancies.

Those inequalities confirm the well-known in cryptography and Information Theory fact that reduction of the redundancy improves the safety of ciphers.

Let us return to the first question of this note about the Vernam cipher with a biased key sequence. More precisely, let there be a plaintext $X_{1} X_{2} \ldots$, $X_{i} \in\{0,1\}$ and the key sequence $Y_{1} Y_{2} \ldots, Y_{i} \in\{0,1\}$, generated by a source whose entropy $h(Y)$ is less then 1 . $\left(h(Y)=1\right.$ if and only if $Y_{1} Y_{2} \ldots$ generated by the Bernoulli source with letter probabilities $P(0)=P(1)=0.5,[2$, 3] $)$. From (3) we can see that the size of the set $\Psi(Z)$ of high-probable possible decipherings grows exponentially with exponent grater than $h(X)-r_{Y}$, where $r_{Y}=1-h(Y)$. So, if $r_{Y}$ goes to 0 , the size of the set of possible probable decipherings trends to the size of this set for the case of "pure" Vernam cipher. Indeed, if $h(Y)=1$ and, hence, $r_{Y}=0$, the set $\Psi(Z)$ of high-probable possible decipherings grows exponentially with exponent $h(X)$, as it should be for the Vernam cipher. For example, it is true for the case where the key sequence $Y_{1} Y_{2} \ldots$ is generated by the Bernulli source with biased probabilities, say $P(0)=0.5-\tau, P(1)=0.5+\tau$, where $\tau$ is a small number. If $\tau$ goes to 0 , the redundancy $r_{Y}$ goes to 0 , too, and we obtain the Vernam cipher. So, we can informally say that the Vernam cipher is robust to small deviations from randomness. 


\section{References}

[1] R. Ahlswede. Remarks on Shannons secrecy systems. Probl. of Control and Inf. Theory, 11(4), 1982, 301-308.

[2] T. M. Cover and J. A. Thomas. Elements of information theory, WileyInterscience, New York, NY, USA, 2006.

[3] R. G. Gallager. Information Theory and Reliable Communication, John Wiley \& Sons, New York, 1968.

[4] M. Hellman. An extension of the Shannon theory approach to cryptography. IEEE Transactions on Information Theory, V. 23 , 31977 , pp. 289 - 294

[5] U. Maurer. Information-Theoretic Cryptography. Advances in Cryptology CRYPTO '99, Lecture Notes in Computer Science, Springer-Verlag, vol. 1666, pp. 4764, 1999.

[6] A. Russell, H. Wang. How to Fool an Unbounded Adversary With a Short Key. IEEE Transactions on Information Theory, VOL. 52, NO. 3, MARCH 2006

[7] B. Ryabko, A. Fionov. Basics of Contemporary Cryptography for IT Practitioners. World Scientific Publishing Co., 2005.

[8] B. Ryabko, D. Ryabko. Confidence Sets in Time - Series Filtering, In: Proceedings of 2011 IEEE International Symposium on Information Theory (ISIT'11), July, 31 - August, 5, 2011, Saint-Petersburg, Russia

[9] Shannon, C. E. (1949). Communication theory of secrecy systems. Bell system technical journal, 28(4), 656-715. 